\title{
Research on Retailer Pricing Based on Extended Regret Theory in E-commerce Environment
}

\author{
Qiu Ge \\ (School of Transportation and Logistics, Southwest Jiaotong University, Chengdu 610031, China; qiuge@ swjtu.cn)
}

\begin{abstract}
Based on the Expected Utility Theory and Regret Theory, the Extended Regret Theory (ERT) is proposed in this paper to study the optimal pricing strategy of retailers in e-commerce environment. Taking the diversity of sales channels and the uncertainty of consumers in e-commerce environment into consideration, author of the paper designs an extended regret utility function which comprehensively considers both pessimistic and optimistic attitudes of decision makers in retailing industry to describe their regret-avoidance behavior. According to the sensitivity analysis, it is found that the optimal retail price decreases as the consumer price sensitivity coefficient increases, yet does not show variation with changes of the consumers pessimism degree. Moreover, the optimal retail price(s) obtained under EUT, ERT and combination of EUT and ERT represent the same.
\end{abstract}

Keywords: Retailers' Optimal Pricing Strategy; Expected Utility Theory (EUT); Regret Theory; Regret Reference Point; Price-dependent Demand

\section{Introduction}

The extensive application of Internet and mobile devices has led to the explosive development of e-commerce and great changes in the way retailers sell products. Combination of online sales and offline distribution has become the main choice of retailers. And the development of optimal pricing strategy has long been a key concern for supply chain managers and researchers. For pricing strategies under different sales channels, Gan et a ${ }^{[1]}$ studied the pricing of new and remanufactured products under separate sales channels and conducted an empirical analysis of the pricing strategy; There's also researchers ${ }^{[2-3]}$ studying the pricing problem of different sales channels under different circumstances. However, few of them in the circle takes notice of the influence of decision makers and decision theory on pricing decisions under different sales channels. This paper proposes the ERT model to describe the regret avoidance behavior of decision makers in e-commerce environment.

EUT believes that the decision makers are completely rational and the rationality of decision makers in its model is reflected in decision makers' expectation based on the expected utility function ${ }^{[4]}$, and the objective function of decision is to maximize the expected utility. However, in real life, given that most decision makers are not completely rational, the optimal pricing strategy of retailers solely based on EUT deviates from the actual supply chain pricing decision ${ }^{[5]}$. In response to that, the prospect theory ${ }^{[6]}$ is developed to quantify the extent to which decision makers are affected by risk preference in the supply chain decision-making process. However, in the process of real optimal pricing decision-making, decision makers are affected not only by risk preference, but could 
also by regrets. Regret is a kind of negative emotion that decision makers increasingly experience in life, and is mostly triggered by the decision makers' imagination after comparing the results of the unchosen decision-making schemes with that of the already done ones. Zhang Shunming and Ye Jun ${ }^{[7]}$, as well as Li Haijun and Xu Fuming et al. ${ }^{[8]}$ pointed out respectively that in behavioral decision-making process, regrets emphasize the existence of "repent function" caused by not choosing a different decision-making scheme. It is believed that each decision will produce an opposite (selecting another) connection and will be compared with the current decision. If the current decision presents a better possible outcome than that of another unchosen one, it provides the decision maker a psychological feeling of gain, otherwise providing a feeling of loss. Regret Theory (RT) was first proposed by Bell (1982). ${ }^{[9]}$ it is later pointed out that the single-factor utility function cannot well describe the irrational behavior of decision-makers. Therefore, factors such as regret and satisfaction are introduced to the utility function.

RT is widely used in various fields because it reveals the psychological changes in decision making ${ }^{[10-13]}$. However, few study approaches the pricing and purchasing strategy of supply chain by taking advantage of RT. Based on EUT and RT, this paper first determines the reference points of decision makers' regrets in e-commerce environment in light of their pessimistic and optimistic attitudes, and then introduces ERT based on regret and satisfaction. In this theory, the exponential function is used to quantify the effectiveness of decision makers based on the reference point of regret emotions. Different positive utility indexes and negative utility indexes are used to quantify the effects of satisfaction and regret on decision makers. Compared with the traditional RT utility function, author of the paper does not solely refer to the reference point of regret emotions when determining the result of the best decision scheme after event, but also the degree of pessimism and optimism of the decision maker. At the same time, the optimal pricing model based on ERT proposed in this paper not only can effectively reflect the bounded rational behavior characteristics of retailers' decision-making in e-commerce environment, but also reflect the influence of decision makers' regret emotions and thus enjoying a strong practical application prospect.

\section{Problem explanation and model development}

\subsection{Questions}

In the process of supply chain operation, retailers' pricing strategy is directly related to the sales volume of products and the overall profits. However, in the case of e-commerce, the uncertainty of consumers increases while the distribution channels of supply chain diversify. As the proposer of the optimal pricing strategy, retailers are subject to certain characteristics of bounded rational actor. Therefore, it is not very practical to use the expected utility theory framework alone to describe retailers' decision-making behavior. Whether the retailer's regret will also affect the formulation of its optimal strategy is also a question worth studying. In order to study impact of changes in supply chain environment on decision makers' pricing decision in e-commerce environment, this paper proposes a retailer's pricing decision model based on the extended regret theory, in the hope of providing effective theoretical and model support for practically optimizing retailers' pricing decisions in e-commerce environment.

\subsection{Symbolic explanation and model assumptions}

For the convenience of explanation, the wholesale price of unit product is referred to as $w$, retail price as $p$, order quantity as $q$, actual market demand of product as $D$ and product residue value as $b$. At the same time, the following model hypothesis is given:

Hypothesis (1): According to the scope of customer perceived utility set by Akcura et $a^{[14]}$, this paper assumes 
that the price range of the product is $p \in\left[p_{L}, p_{H}\right] ; p_{L}$ denotes the lowest price a retailer can accept for a product while $p_{H}$ denotes the highest price a consumer can accept for a product.

Hypothesis (2): The initial market demand of the product is $X$, subject to uniform distribution and normal distribution, etc. In order to show the relationship between the optimal pricing and purchasing strategy with the corresponding parameters, it is assumed that $X$ is subject to uniform distribution alone, i.e. $X \sim U\left(\mu_{1}, \mu_{2}\right)$

Considering the price dependence of product market demand ${ }^{[15]}$, we set the market demand as below:

$$
D=\lambda(p) X
$$

Wherein, $\lambda(p)=a p^{-\delta}$ is the product price dependence function ${ }^{[16]} ; a$ is a constant and $a>0$ indicates the consumers' perception of the price of the product; $\delta$ refers to the price sensitivity coefficient of consumers affected by the price of the product, $\delta \geq 1$, and the larger the value of $\delta$, the stronger the consumer is affected by the product price; $X$ is a random variable representing the initial market demand of the product. $f(X)$ and $F(X)$ represent respectively the probability density function and the cumulative probability distribution function of $X$. Assuming random variable $D$ is the actual market demand of the product, and probability density function and cumulative probability distribution function of which are $g(D)$ and $G(D)$ respectively. Therefore, the relationship between the random variable $D$ and $X$ could be shown as:

$$
g(D)=\frac{1}{\lambda(p)} f(X)
$$

\subsection{Expected utility model}

When the order quantity $q$ of the product is greater than its actual market demand $D$, the expected sales volume of the product is:

$$
\begin{aligned}
E^{1}(q) & =\int_{0}^{q} D g(D) \mathrm{d} D \\
& =\int_{0}^{q / \lambda}(p)[\lambda(p) X f(X) / \lambda(p)] \mathrm{d} \lambda(p) X \\
& =q F(q / \lambda(p))-\lambda(p) \int_{0}^{q / \lambda(p)} F(X) \mathrm{d} X
\end{aligned}
$$

At this point, the expected surplus amount of the product is:

$$
\begin{aligned}
\hat{E}(q) & =\int_{0}^{q}(q-D) g(D) \mathrm{d} D \\
& =\int_{0}^{q / \lambda(p)}(q-\lambda(p) X) f(X) \mathrm{d} X \\
& =q F(q / \lambda(p))-E^{1}(\pi)
\end{aligned}
$$


When the order quantity $q$ of the product is smaller than or equal to the market demand $D$, the expected sales volume of the product is:

$$
\begin{aligned}
E^{2}(q) & =\int_{q}^{+\infty} q g(D) \mathrm{d} D \\
& =\int_{q / \lambda(p)}^{+\infty}[q f(X) / \lambda(p)] \mathrm{d} \lambda(p) X \\
& =q(1-F(q / \lambda(p)))
\end{aligned}
$$

The expected sales volume obtained by combining formula (3) and (5) is:

$$
\begin{aligned}
E(q)= & E^{1}(q)+E^{2}(q) \\
= & q F(q / \lambda(p))-\lambda(p) \int_{0}^{q / \lambda(p)} F(X) \mathrm{d} X \\
& +q(1-F(q / \lambda(p))) \\
= & q-\lambda(p) \int_{0}^{q / \lambda(p)} F(X) \mathrm{d} X
\end{aligned}
$$

Therefore, the expected profit of the retailer obtained from formula (4)- (6) is:

$$
\begin{aligned}
E\left(\pi_{r}\right) & =p E(q)-w q+b \hat{E}(q) \\
& =(p-w) q+(b-p) \lambda(p) \int_{0}^{q / \lambda(p)} F(X) \mathrm{d} X
\end{aligned}
$$

According to Hypothesis(2), when $X \sim U\left(\mu_{1}, \mu_{2}\right)$, the retailer's expected profit function is:

$$
\begin{gathered}
E\left(\pi_{r}\right) \\
=\left\{\begin{array}{lr}
(p-w) q, & X<\mu_{1} ; \\
\frac{\lambda(p)(b-p)}{\mu_{2}-\mu_{1}}\left[\frac{q}{\lambda(p)} \mu_{2}-\frac{q^{2}}{2 \lambda^{2}(p)}-\mu_{1} \mu_{2}+\frac{\mu_{1}^{2}}{2}\right] \\
+(p-w) q, & \mu_{1} \leq X \leq \mu_{2} ; \\
(b-w) q+\frac{\left(\mu_{1}+\mu_{2}\right) \lambda(p)}{2}(p-b), & X>\mu_{2} .
\end{array}\right.
\end{gathered}
$$

Proposition 1: When $X \sim U\left(\mu_{1}, \mu_{2}\right)$, the retailer's expected profit $E\left(\pi_{r}\right)$ is a concave function of the retail price $p$, and there exists an optimal retail price $p_{E U T}$ to maximize the retailer's expected profit.

Proof: Solve the first and second partial derivatives of $E\left(\pi_{r}\right)$ respectively by retail price $p$ and order quantity $q$ for formula (8). 


$$
\begin{gathered}
\mathrm{d} E\left(\pi_{r}\right) / \mathrm{d} p \\
=\left\{\begin{array}{rr}
q, & p<\sqrt[\delta]{a \mu_{1} / q} ; \\
q+\frac{q}{\mu_{2}-\mu_{1}}\left[\delta\left(1-b p^{-1}\right)\left(1+\mu_{1}-a^{-1} p^{\delta} q\right)-1\right], & \sqrt[\delta]{a \mu_{1} / q} \leq p \leq \sqrt[\delta]{a \mu_{2} / q} ; \\
a \mu_{1} p^{-\delta}\left(1-\delta+b p^{-1} \delta\right), & p>\sqrt[\delta]{a \mu_{2} / q} .
\end{array}\right. \\
\frac{\mathrm{d}^{2} E\left(\pi_{r}\right)}{\mathrm{d} p^{2}}=\left\{\begin{array}{rr}
0, & \sqrt[\delta]{a \mu_{1} / q} \text { 或 } p>\sqrt[\delta]{a \mu_{2} / q} ; \\
-\frac{q^{2} a^{-1} \delta p^{\delta-1}}{\mu_{2}-\mu_{1}}\left[\frac{\delta+1}{2}+b p^{-1}(\delta-1)\right], & \sqrt[\delta]{a \mu_{1} / q} \leq p \leq \sqrt[\delta]{a \mu_{2} / q} .
\end{array}\right.
\end{gathered}
$$

Since $Q>0, \delta>1$ and $\mu_{2}>\mu_{1}$, according to formula (9), when the retail price of the product $p<\sqrt[\delta]{a \mu_{1} / Q}$, $\mathrm{d} E\left(\pi_{r}\right) / \mathrm{d} p>0$, the expected profit of the retailer $E\left(\pi_{s}\right)$ monotonically increases with respect to $p$; when $p>\sqrt[\delta]{a \mu_{2} / Q}, \mathrm{~d} E\left(\pi_{r}\right) / \mathrm{d} p<0$, then $E\left(\pi_{s}\right)$ decreases monotonically with respect to $p$; and given that $E\left(\pi_{s}\right)$ is a continuous function of the retail price $p$, the retailer's expected profit has a maximum point. At the same time, in formula (10), when $\sqrt[\delta]{a \mu_{1} / Q} \leq p \leq \sqrt[\delta]{a \mu_{2} / Q}, \frac{\mathrm{d}^{2} E\left(\pi_{r}\right)}{\mathrm{d} p^{2}}<0$, it is indicated that the expected profit of the retailer is a concave function of the retail price $p$. Therefore, when $\sqrt[\delta]{a \mu_{1} / Q} \leq p \leq \sqrt[\delta]{a \mu_{2} / Q}$, there exits the optimal retail price $p_{E U T}$ that maximizes the expected profit of the retailer, thus Proposition 1 is proved.

Proposition 2: When $X \sim U\left(\mu_{1}, \mu_{2}\right)$, retailer's expected profit $E\left(\pi_{r}\right)$ is a monotonic decreasing function of consumer price sensitivity coefficient $\delta$.

Proof: Solve the first and second derivatives of formula (7) for consumer price sensitivity coefficient $\delta$

$$
\frac{\mathrm{d} E\left(\pi_{r}\right)}{\mathrm{d} \delta}=\left\{\begin{array}{rr}
0, & p<\sqrt[\delta]{a \mu_{1} / Q} ; \\
-\frac{\delta \lambda(p)}{2\left(\mu_{2}-\mu_{1}\right)}\left(\frac{Q^{2}}{\lambda^{2}(p)}\left(\frac{2 b}{p}+1\right)-\mu_{1}^{2}\right) & \sqrt[\delta]{a \mu_{1} / Q} \leq p \leq \sqrt[\delta]{a \mu_{2} / Q} \\
-2 \delta \lambda(p) \mu_{2} \frac{\mu_{1}+\mu_{2}}{2}, & p>\sqrt[\delta]{a \mu_{2} / Q} .
\end{array}\right.
$$

In the above formula, when $\sqrt[\delta]{a \mu_{1} / Q} \leq p \leq \sqrt[\delta]{a \mu_{2} / Q}$ and $\mu_{1}<Q / \lambda(p), \mu_{1}^{2}<Q^{2} / \lambda^{2}(p)$, so $\mathrm{d} E\left(\pi_{r}\right) / \mathrm{d} \delta<0$; when $p>\sqrt[\delta]{a \mu_{2} / Q}$, then $\mathrm{d} E\left(\pi_{r}\right) / \mathrm{d} \delta<0$. Therefore, retailer's expected profit $E\left(\pi_{r}\right)$ is monotone decreasing function of consumer price sensitivity coefficient $\delta$. Proposition 2 is proved.

The optimal retail price under the circumstance when the retailer expects to maximize profit can therefore be solved by $\mathrm{d} E\left(\pi_{r}\right) / \mathrm{d} p=0$ in formula (9): 


$$
M=(\delta+1) p^{\delta} / 2+b \delta p^{\delta-1}
$$

In the above formula, $M=a\left(\mu_{2}-2 \delta \mu_{1}\right) / Q$. In formula (11), when the retailer's price sensitivity coefficient $\delta$ varies, the optimal retail price obtained by the retailer becomes different. For example, when $\delta=1, p_{E U T}=M-b$; but when $\delta=2, \quad p_{E U T}=\left(\sqrt{b^{2}+6 M}-b\right) / 3$. Therefore, the optimal retail price is affected by such factors as the distribution of product market demand, order volume, consumer price perception constant, consumer price sensitivity coefficient, etc.

To solve the maximum and minimum expected profits of the retailer, we can see from theorem 1 that :

$$
\begin{aligned}
\text { when } & p_{E U T} \in\left[p_{L}, p_{H}\right], \\
\max _{p \in\left[p_{L}, p_{H}\right]} E\left(\pi_{r}\right) & =E\left(\pi_{r}\left(p_{L}\right)\right) \\
\min _{p \in\left[p_{L}, p_{H}\right]} E\left(\pi_{r}\right) & =\min \left\{E\left(\pi_{r}\left(p_{L}\right)\right), E\left(\pi_{r}\left(p_{H}\right)\right)\right\} \\
\text { when } p_{E U T} \notin\left[p_{L}, p_{H}\right], & \\
\max _{p \in\left[p_{L}, p_{H}\right]} E\left(\pi_{r}\right) & =\max \left\{E\left(\pi_{r}\left(p_{L}\right)\right), \quad E\left(\pi_{r}\left(p_{H}\right)\right)\right\} \\
\min _{p \in\left[p_{L}, p_{H}\right]} E\left(\pi_{r}\right) & =\min \left\{E\left(\pi_{r}\left(p_{L}\right)\right), E\left(\pi_{r}\left(p_{H}\right)\right)\right\}
\end{aligned}
$$

The retail prices at this point are the prices at which the retailer gains the maximum or minimum profit.

\subsection{Extended regret utility model}

Quiggin (1994) [17] and Sugden (1993) [18] put forward the axiomatic regret theory, using $\hat{H}(U(t)-U(s))$ to represent the utility (regret utility function) generated by decision maker's regret emotions. It is a function satisfying $\hat{H}(0)=0, \hat{H}(0)=0$, in which $t$ and $s$ represent the two decision-making strategies of the decision maker respectively. Specifically, $U(t)$ represents the actual expected utility of the decision maker while $U(s)$ the reference point of regret utility of the decision maker after event.

In the existing research, most researchers take the best afterwards decision result of the decision-maker as the reference point of regret emotions, where $\hat{H}(U(t)-U(s)) \leq 0$ always holds. When $U(t)=U(s)$, the decisionmaker's scheme $t$ and the best decision-making scheme ${ }^{s}$ are the same, and only under this circumstances, the decision makers will not produce negative effect because of regret emotions, and such function is called the traditional regret utility function (as marked by dash line in figure 1). However, in the practical decision-making process, the decision maker does not always take the best decision outcome after event as the reference point of regret. For example, when the decision maker is always optimistic about life, he may take the industry average income as a reference point for actual decision-making results. In such case, the regret utility function produces 
positive value, thus the traditional regret utility function fails to demonstrate the effect of positive utility on decision makers.

Therefore, this paper establishes an optimal pricing model based on RT according to the degree of pessimism and optimism in the optimal pricing decision of retailers. In the process of model establishment, the linear combination of the two extremes of the two attitudes (absolute pessimist and absolute optimist) was used to represent the reference point of decision makers' regret emotions and thus replace the reference point of traditional regret emotions (the best decision result after event $U(s)$ ).In the process of optimal pricing decision of supply chain, absolute pessimists always take the maximum expected profit as the reference point of regret. If the final return can reach the reference point, the retailer will be satisfied, otherwise the utility function of regret will produce negative utility. Meanwhile, absolute optimists always take the minimum expected profit as the reference point for regret. If the final benefit can be greater than the reference point, the retailer will be satisfied and the regret utility function will produce positive utility. In this paper, we determine the regret emotion reference point $r$ in light of the pessimistic and optimistic level of the decision maker. Assuming that the optimal decision scheme and the worst decision scheme are $s$ and $m$ respectively, the expected utility values are $U(s)$ (maximum expected profit) and $U(m)$ (minimum expected profit) correspondingly, thus the reference point of regret utility can be defined as following:

$$
\begin{aligned}
U(r) & =\beta U(s)+(1-\beta) U(m) \\
& =\beta \max _{p \in\left[p_{L}, p_{H}\right]} E\left(\pi_{r}\right)+(1-\beta) \min _{p \in\left[p_{L}, p_{H}\right]} E\left(\pi_{r}\right)
\end{aligned}
$$

In this formula, $\beta \in[0,1]$ and it represents the retailer's pessimism coefficient, describing the degree of pessimism. The bigger the value of $\beta$ is, the more pessimistic the retailer is. When $\beta=0$, the retailer is an absolutely optimistic while when $\beta=1$, absolutely pessimistic, and in such case, the regret utility function is equal to the traditional regret utility function. Based on the reference point of regret utility defined above and according to its nature (reference NO. [9] and NO. [10]), the new regret utility function is defined as follows:

$$
\begin{aligned}
& H(U(t)-U(r)) \\
= & \left\{\begin{array}{cc}
-\eta[-(U(t)-U(r))]^{\xi}, & U(t)<U(r) ; \\
0, & U(t)=U(r) ; \\
{[U(t)-U(r)]^{\rho},} & U(t)>U(r) .
\end{array}\right.
\end{aligned}
$$

In the formula, $\xi$ and $\rho$ respectively represent the negative utility index and positive utility index of the decision maker's regret utility, and $0<\xi, \rho<1 ; \xi \neq \rho$ indicates the different degree of influence on the decision maker by post-event regret or satisfaction. The larger $\xi$ or $\rho$ is, the greater the influence of the post-event regret or satisfaction is, and vice versa. $\eta \geq 1$ is the negative utility coefficient of the regret utility, indicating that the regret emotions generated through the regretful utility function has a greater impact on the decision-maker than that of satisfaction when the maximum expected profit after event is less than the regret reference point. At the same time, it is also noted that when the value obtained through formula (16) is positive, the regret utility produces positive utility and the decision-maker feels happy. The larger the value is, the greater the degree of satisfaction is. 
On the contrary, it means that the regret utility produces negative utility, and the decision maker feels disappointed, and the smaller the value, the greater the regret degree. Therefore, the model of formula (16) quantifies the emotional changes of decision makers, either in terms of regret or satisfaction based on the regret utility function, enabling a vivid description of the decision-making process.

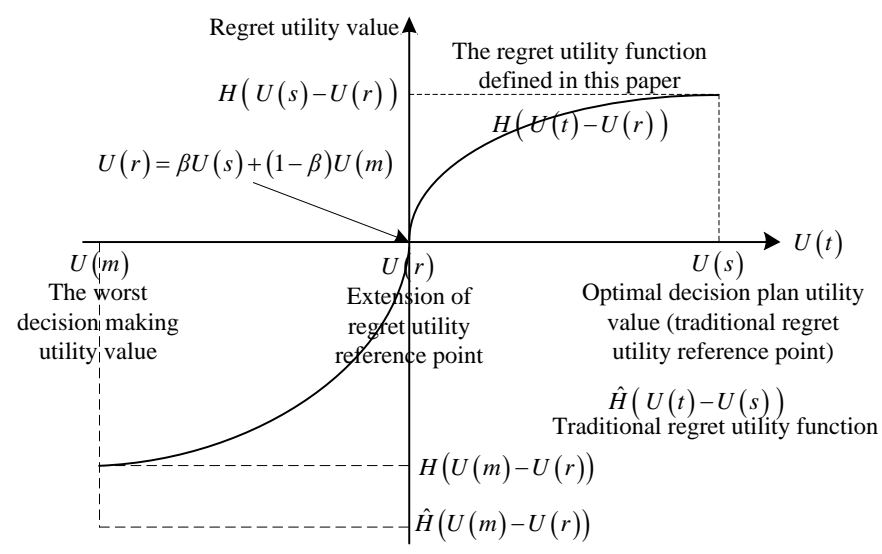

Figure 1 Comparison between the traditional regret utility function and the regret utility function introduced in this paper

The solid line in Figure 1 shows the trend of the new regret utility function introduced in this paper. It can be seen that when the decision scheme is the optimal decision scheme after event, the utility value of the traditional utility function (as marked by dash line in the figure) is 0 , under which circumstances the value of the utility function defined in this paper is $H(U(s)-U(r))$. At this point, the decision maker is pleased with his initial choice and $U(r)$ is defined as formula (14). When the decision scheme is the worst decision scheme $m$ after event, the utility value of the traditional regret utility function is $\hat{H}(U(m)-U(s))$, and the utility value of the new regret utility function defined in this paper is $H(U(m)-U(r))$.

Theorem 3: According to formula (16), the regret utility function introduced in this paper satisfies $H(0)=0$, and when $U(t) \neq U(r)$, then $H^{\prime}(Y)>0$, where $Y=U(t)-U(r)$.

Proof: $H(0)=0$ is clearly established. Given that $0<\xi, \rho<1$, then when $U(t)<U(r)$ or when $Y<0$, $H^{\prime}(Y)=\xi(-Y)^{\xi-1}>0$; when $U(t)>U(r)$, or when $Y>0, H^{\prime}(Y)=\rho Y^{\rho-1}>0$, the theorem is thus proved.

Based on the reference point defined by formula (14), the following optimal pricing model based on regret theory can thus be established:

$$
\max _{p \in\left[p_{L}, p_{H}\right]} H\left\{E\left(\pi_{r}\right)-\left[\begin{array}{l}
\beta \max _{p \in\left[p_{L}, p_{H}\right]} E\left(\pi_{r}\right) \\
+(1-\beta) \min _{p \in\left[p_{L}, p_{H}\right]} E\left(\pi_{r}\right)
\end{array}\right]\right\}
$$

In the formula, $\max _{p \in\left[p_{L}, p_{H}\right]} E\left(\pi_{r}\right)$ and $\min _{p \in\left[p_{L}, p_{H}\right]} E\left(\pi_{r}\right)$ respectively represent the maximum and minimum 
expected profits of the retailer, and value of which can be obtained through formula (13) and (14) respectively. The regret utility function $H(\cdot)$ is defined as shown in formula (14).

When $H(\cdot)>0$, that is,

$$
E\left(\pi_{r}\right)>\beta \max _{p \in\left[p_{L}, p_{H}\right]} E\left(\pi_{r}\right)+(1-\beta) \min _{p \in\left[p_{L}, p_{H}\right]} E\left(\pi_{r}\right)
$$

The retailer is satisfied because the actual result value obtained is greater than the reference point, and then the regret utility function produces positive utility value;

On the contrary, when $H(\cdot)<0$, that is,

$$
E\left(\pi_{r}\right)<\beta \max _{p \in\left[p_{L}, p_{H}\right]} E\left(\pi_{r}\right)+(1-\beta) \min _{p \in\left[p_{L}, p_{H}\right]} E\left(\pi_{r}\right)
$$

The retailer is disappointed and regretful because the actual result value obtained is smaller than the expected reference point, and then the regret utility function produces negative utility value.

Theorem 4: Under RT, the retailer's regret utility value $H(\cdot)$ decreases as the pessimistic coefficient $\beta$ increases.

Proof: To facilitate illustration, the utility function $H\left\{E\left(\pi_{r}\right)-\left[\beta \max _{p \in\left[p_{L}, p_{H}\right]} E\left(\pi_{r}\right)+(1-\beta) \min _{p \in\left[p_{L}, p_{H}\right]} E\left(\pi_{r}\right)\right]\right\}$ in formula (17) is abbreviated as $H(\cdot)$, and the first derivative of the utility function with respect to the pessimistic coefficient $\beta$ is as follows:

$$
\frac{\mathrm{d} H(\cdot)}{\mathrm{d} \beta}=H^{\prime}(\cdot)\left[\min _{p \in\left[p_{L}, p_{H}\right]} E\left(\pi_{r}\right)-\max _{p \in\left[p_{L}, p_{H}\right]} E\left(\pi_{r}\right)\right]
$$

According to theorem 3, $H^{\prime}(\cdot)>0$, and because $\max _{p \in\left[p_{L}, p_{H}\right]} E\left(\pi_{r}\right)>\min _{p \in\left[p_{L}, p_{H}\right]} E\left(\pi_{r}\right)$, thus $\mathrm{d} H(\cdot) / \mathrm{d} \beta<0$. In response to that, the regret utility value $H(\cdot)$ of the retailer decreases with the increase of the pessimistic coefficient $\beta$. Theorem 4 is thus proved.

From the practical aspects, the retailer's pessimistic coefficient $\beta$ reflects the pessimism degree of the retailer. The larger the value of $\beta$ is, the more pessimistic the retailer is, the more useful the reference point is, and the less satisfied the retailer is with the actual outcome because of the smaller regret utility.

Theorem 5: The optimal price of retailer $p_{R T}$ under RT is equal to the optimal price of retailer $p_{E U T}$ under EUT.

Proof: For convenience of illustration, let 


$$
Y=E\left(\pi_{r}\right)-\left[\beta \max _{p \in\left[p_{L}, p_{H}\right]} E\left(\pi_{r}\right)+(1-\beta) \min _{p \in\left[p_{L}, p_{H}\right]} E\left(\pi_{r}\right)\right]
$$

then, it can be obtained through formula (17) that the utility function of regret is $H(Y)$. According to theorem 1 , there exits the optimal retail price $p_{E U T}$ under EUT that maximizes the retailer's expected profit

$$
E\left(\pi_{r}\left(p_{E U T}\right)\right)=\max E\left(\pi_{r}(p)\right)
$$

That is, $\mathrm{t} p_{E U T}$ is the maximum value of $Y$.From formula (16) as a supplement, given that $H(Y)$ is a monotonic increasing function of $Y$, then

$$
H\left\{E\left(\pi_{r}\left(p_{E U T}\right)\right)\right\}=\max H\left\{E\left(\pi_{r}(p)\right)\right\}
$$

So $p_{R T}=p_{E U T}$. Theorem 5 is thus proved.

\subsection{Optimal pricing decision model based on ERT}

In the process of price-making of retailers in e-commerce environment, the decision-making subject is the person with bounded rationality. The decision-maker not only asks for the maximum expected profit, but also subconsciously considers the regret factor before decision-making, that is, trying to avoid regret. (When the regret utility function produces a negative value, the decision-makers wants to minimize the negative effect while in the case of positive value, maximize the positive effect). Therefore, taking the behavioral characteristics of retailers in decision-making into consideration, this paper establishes the following double-objective function:

$$
\left\{\begin{array}{l}
\max _{p \in\left[p_{L}, p_{H}\right]} E\left(\pi_{r}\right) \\
\max _{p \in\left[p_{L}, p_{H}\right]} H\left\{E\left(\pi_{r}\right)-\left[\beta \max _{p} E\left(\pi_{r}\right)+(1-\beta) \min _{p} E\left(\pi_{r}\right)\right]\right\}
\end{array}\right.
$$

The target constraint is:

$$
E\left(\pi_{r}\right)=(p-w) Q+(b-p) \lambda(p) \int_{0}^{Q / \lambda(p)} F(X) \mathrm{d} X ;\left\{\begin{array}{l}
p_{L} \leq p \leq p_{H}, 0 \leq \beta \leq 1,0<\xi, \rho<1 \\
w \geq b \geq 0 ; Q, a \geq 0 ; \delta \geq 1 \\
\lambda(p)=a p^{-\delta}
\end{array}\right.
$$

Wherein $E\left(\pi_{r}\right)$ represents the expected utility function of the retailer; $H(\cdot)$ the regret utility function of the retailer, which depends on the expected utility reference point of the retailer after the product is sold and the actual decision result. In order to reflect the influence of the regret emotions on the decision-making of the retailer, the two-objective function of formula (17) is transformed into the following single-objective function for solving: 


$$
\begin{aligned}
& \max _{p \in\left[p_{L}, p_{H}\right]} S \\
& S=(1-\kappa) E\left(\pi_{r}\right)+ \\
& \kappa H\left\{E\left(\pi_{r}\right)-\left[\beta \max _{p \in\left[p_{L}, p_{H}\right]} E\left(\pi_{r}\right)+(1-\beta) \min _{p \in\left[p_{L}, p_{H}\right]} E\left(\pi_{r}\right)\right]\right\}
\end{aligned}
$$

wherein, $\kappa \geq 0$ is the regret coefficient; when $\kappa=0$, formula (21) is $\max _{p} E\left(\pi_{r}\right)$, the expected utility function, indicating that the retailer's decision is the decision of a fully rational person. $\kappa>0$ indicates that the retailer is affected by the regret emotions.

Theorem 6: It can be deduced from formula (21) that when $E\left(\pi_{r}\right)>H(\cdot)$, the single objective utility function $S$, transformed from the double objective of expected utility and regret utility function is the monotonic decreasing function of the retailer's regret coefficient $\kappa$; when $E\left(\pi_{r}\right)<H(\cdot)$, the single objective utility function $S$ is the monotonic increasing function of the retailer's regret coefficient $\kappa$; and when $E\left(\pi_{r}\right)=H(\cdot)$, the single objective utility function $S$ is equal to the expected utility and the regret utility function.

\section{Sensitivity analysis}

Suppose a retailer sells a certain type of electronic component, and corresponding parameters $c=35, w=50$, $X \rightarrow U(0,12), a=80, p_{L}=60, p_{H}=160$ and $b=30$.

It can be concluded from formula (21) that different regret coefficient $\kappa$ reflects different regret degree of the retailer. Here, we first assume that $\kappa=0.40$, and parameters of regret utility function are set as follows: $\xi=0.85$, $\rho=0.88$ and $\eta=1.00$. The relationship between retailer utility and product retail price based on EUT and RT is thus shown in figure 2 and 3.

Figure 2 shows the correlation between retailer utility value and retail price under different consumer price sensitivity coefficients. It is found that as the consumer price sensitivity increases, the optimal retail price and the retailer's optimal utility decreases. Moreover, the price sensitivity coefficient of consumers exerts a great influence on the optimal pricing of products and the utility value of retailers. When $\delta$ increases by $5.88 \%$, the optimal price decreases by $32.46 \%$ and the retailer utility value reduces by $58.38 \%$. For this reason, in the practical pricing strategy formulation, it is necessary to carry out market investigations to analyze the price sensitivity coefficient of the consumer, and the results of which are directly related to the retailer's optimal pricing and determine profitability and profit margins of the retailer. 


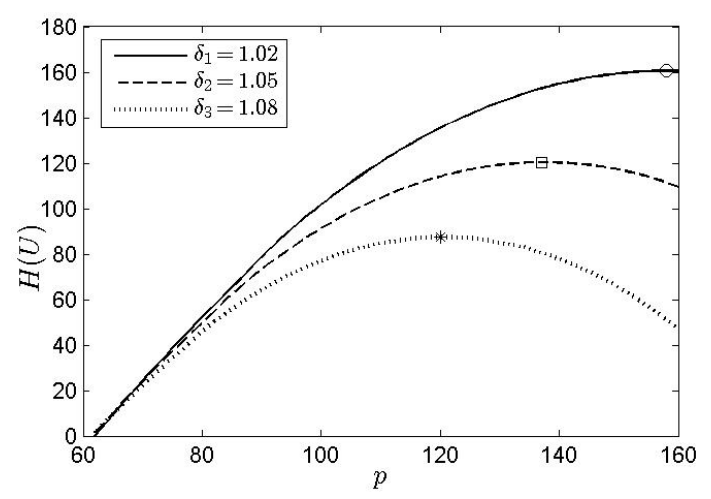

Fig. 2 Correlation between retailer utility value and product retail price under different consumer price sensitivity coefficients $\delta$

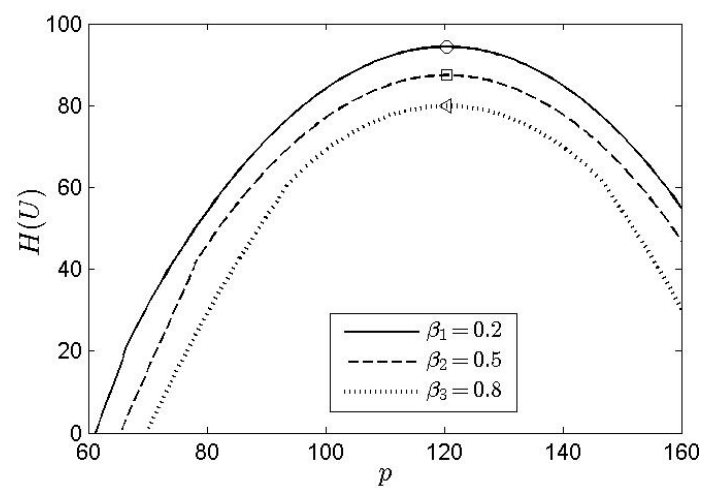

Fig.3 Correlation between retailer utility value and product retail price under the pessimistic coefficients of different retailers $\beta$

Figure 3 shows the correlation between the retailer's utility value and the retail price when the retailer's pessimistic coefficient $\beta$ increases and the consumer's price-sensitive coefficient is determined. It can be concluded from figure 3 that the more pessimistic the retailer is, that is, the larger the value of $\beta$ is, the smaller the utility value is, yet the optimal product retail price is not affected by it. Provided that the change of the value of $\beta$ leads to variation of the reference point of the retailer's regret alone, and the reduction of the regret utility value, the total utility of the retailer thus also decreases.

Figure 4 shows the correlation between retailer utility value and retail price under three theoretical frameworks. In figure 4, the solid line indicates the optimal pricing strategy under the expected utility theory. As the decisionmaker is not affected by any emotional factors in the decision-making process, the retailer's expected utility value under this theoretical framework is the maximum. The dotted line represents the optimal pricing strategy under the regret theory, which approaches the effect of consideration of the after-effects on the retailer in decision-making, where the utility value is small. The line of dashes reflects the correlation between utility value and retail price under the utility function based on both the expectation utility theory and the regret theory. It is found, as shown in the figure that as this utility function incorporates the expected utility and the regret utility, it delivers better performance in reflection the psychological change of the retailer in the process of pricing. At the same time, the curve of figure 4 shows that the optimal retail prices under the three theoretical frameworks are all the same, which coincides with the theorem 5 . 


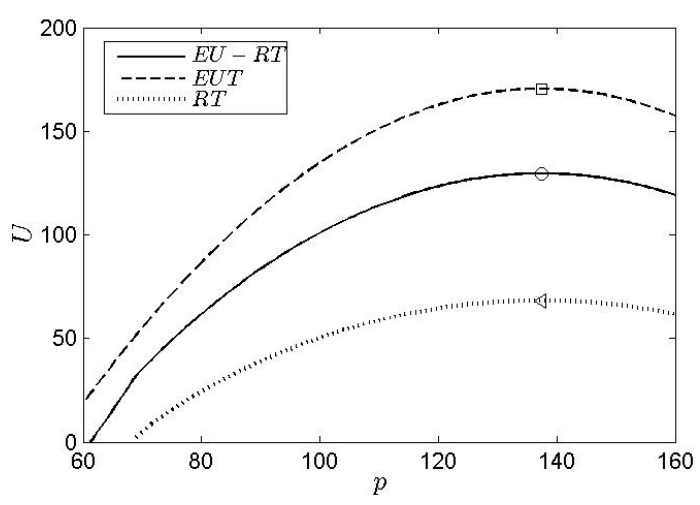

FIG. 4 Correlation between retailer utility value and retail price under different theoretical frameworks

\section{Conclusion}

The rapid development of e-commerce has diversified the supply chain distribution channels and increased the uncertainty of consumer demand. Faced with the complex and ever-changing supply and demand environment, this paper takes advantage of the linear combination of the best and worst schemes to express the regret utility reference point based on the existing expected utility theory and regret theory. In light of the newly introduced regret utility reference point, author of the paper proposes a new regret utility function with power function quantifying the impact of satisfaction and regret of the decision maker, and establishes the retailer's optimal pricing decision model based on the extended regret theory. Meanwhile, the sensitivity analysis is made to demonstrate influences of consumer price sensitivity coefficient, the retailer's pessimism coefficient and different theoretical frameworks on optimal pricing strategies and maximum utility value. The main objective of this paper is to put forward a new regret utility function and establish a multi-objective pricing model in light of the expected utility theory to reflect the bounded rational behavior of the decision-maker in e-commerce and effectively quantify the influence of the regret emotions in retailers' decision-making process, thus providing retailers a reliable and effective theoretical basis in formulation of pricing strategy in e-commerce environment.

\section{References}

[1] Gan S S, Pujawan I N, Widodo B. Pricing decision for new and remanufactured product in a closed-loop supply chain with separate sales-channel[J]. International Journal of Production Economics, 2017, 190: 120-132.

[2] Ding Q, Dong C, Pan Z. A hierarchical pricing decision process on a dual-channel problem with one manufacturer and one retailer[J]. International Journal of Production Economics, 2016, 175: 197-212.

[3] Wu sheng, Chen Zhensong, Li Yanlai, et al. Supply chain pricing decision and sales channel selection based on retailer credit in competitive environment [J]. Computer integrated manufacturing system, 2017, 23(6):1341-1351.

[4] Von Neumann J, Morgenstern O. Theory of games and economic behavior[M]. Princeton University Press, 1944.

[5] Fisher M A, Raman A. Reducing the cost of demand uncertainty through accurate response to early sales [J].Operations Research, 1998, 44(1): 87-99.

[6] Kahneman D, Tversky A. Prospect theory: an analysis of decision under risk[J]. Econometrica, 1979, 7:263-291.

[7] Zhang Shunming, Ye Jun. Review of regret theory [J]. Systems engineering, 2009,2(27): 45-50.

[8] Li Haijun, Xu Fuming, Xiang Peng, et al. Reference dependence based on prediction theory [J]. Psychological science progress, 2013, 2(21):317-325.

[9] Bell D E. Regret in decision making under uncertainty [J].Operations research, 1982, 30: 961-981.

[10] Wei Xiaochao, Li Yanfeng, Chen Donglin. Research on consumer decision-making interaction behavior of new product diffusion based on regret theory and multi-agent simulation [J]. Chinese management science, 2017(11): 66-75. 
[11] Hozo I, Tsalatsanis A, Djulbegovic B. Expected utility versus expected regret theory versions of decision curve analysis do generate different results when treatment effects are taken into account [J]. Journal of evaluation in clinical practice, 2018, 24(1): $65-71$.

[12] Li Meng, Huang Haijun. Research on travel route choice behavior based on regret theory [J]. Journal of management science, 2017, 20(11):1-9.

[13] Trachanas G P, Forouli A, Gkonis N, et al. Hedging uncertainty in energy efficiency strategies: A minimax regret analysis[J]. Operational Research, 2018: 1-16.

[14] Akçura M T, Ozdemir Z D, Rahman M S. Online Intermediary as a Channel for Selling Quality - Differentiated Services[J]. Decision sciences, 2015, 46(1): 37-62.

[15] Venegas B B, Ventura J A. A two-stage supply chain coordination mechanism considering price sensitive demand and quantity discounts[J]. European Journal of Operational Research, 2018, 264(2): 524-533.

[16] Bernstein F, Li Y, Shang K. A simple heuristic for joint inventory and pricing models with lead time and backorders[J]. Management Science, 2015, 8(62): 2358-2373

[17] Quiggin J. Regret theory with general choice sets[J]. Journal of Risk and Uncertainty, 1994, 8(2):153-165.

[18] Sugden R. An axiomatic foundation of regret [J]. Journal of Economic Theory, 1993, 60(6):159-180. 